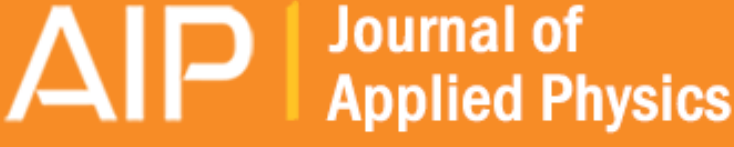

\section{The stochastic dynamics of tethered microcantilevers in a viscous fluid}

Brian A. Robbins, Milad Radiom, William A. Ducker, John Y. Walz, and Mark R. Paul

Citation: Journal of Applied Physics 116, 164905 (2014); doi: 10.1063/1.4900525

View online: http://dx.doi.org/10.1063/1.4900525

View Table of Contents: http://scitation.aip.org/content/aip/journal/jap/116/16?ver=pdfcov

Published by the AIP Publishing

\section{Articles you may be interested in}

Rheology of fluids measured by correlation force spectroscopy

Rev. Sci. Instrum. 83, 043908 (2012); 10.1063/1.4704085

Thermal noise of microcantilevers in viscous fluids

J. Appl. Phys. 104, 104906 (2008); 10.1063/1.3021102

The stochastic dynamics of rectangular and V-shaped atomic force microscope cantilevers in a viscous fluid and near a solid boundary

J. Appl. Phys. 103, 094910 (2008); 10.1063/1.2912989

Transport coefficients of a mesoscopic fluid dynamics model

J. Chem. Phys. 119, 6388 (2003); 10.1063/1.1603721

Stochastic simulations of DNA in flow: Dynamics and the effects of hydrodynamic interactions

J. Chem. Phys. 116, 7752 (2002); 10.1063/1.1466831

MIT LINCOLN

LABORATORY CAREERS

Discover the satisfaction of innovation and service

to the nation
- Space Control

- Air \& Missile Defense

- Communications Systems \& Cyber Security

- Intelligence, Surveillance and

Reconnaissance Systems

- Advanced
Electronics
- Tactical Systems
- Homeland
Protection
- Air Traffic Control

LINCOLN LABORATORY

MassachusetTs Institute of TeChNOLOgY

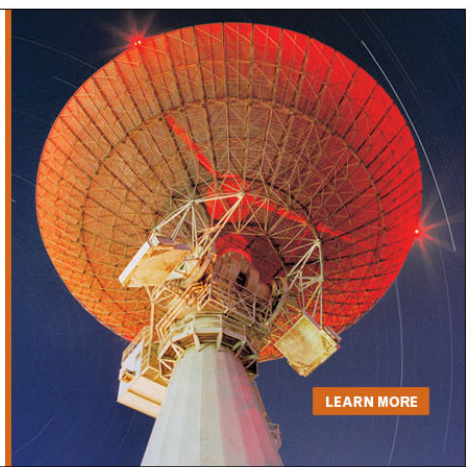




\title{
The stochastic dynamics of tethered microcantilevers in a viscous fluid
}

\author{
Brian A. Robbins, ${ }^{1}$ Milad Radiom, ${ }^{2}$ William A. Ducker, ${ }^{2}$ John Y. Walz, ${ }^{3}$ and Mark R. Paul ${ }^{1}$ \\ ${ }^{1}$ Department of Mechanical Engineering, Virginia Tech, Blacksburg, Virginia 24061, USA \\ ${ }^{2}$ Department of Chemical Engineering, Virginia Tech, Blacksburg, Virginia 24061, USA \\ ${ }^{3}$ Department of Chemical Engineering, University of Kentucky, Lexington, Kentucky 40506, USA
}

(Received 8 August 2014; accepted 15 October 2014; published online 29 October 2014)

\begin{abstract}
We explore and quantify the coupled dynamics of a pair of micron scale cantilevers immersed in a viscous fluid that are also directly tethered to one another at their tips by a spring force. The spring force, for example, could represent the molecular stiffness or elasticity of a biomolecule or material tethered between the cantilevers. We use deterministic numerical simulations with the fluctuation-dissipation theorem to compute the stochastic dynamics of the cantilever pair for the conditions of experiment when driven only by Brownian motion. We validate our approach by comparing directly with experimental measurements in the absence of the tether which shows excellent agreement. Using numerical simulations, we quantify the correlated dynamics of the cantilever pair over a range of tether stiffness. Our results quantify the sensitivity of the auto- and cross-correlations of equilibrium fluctuations in cantilever displacement to the stiffness of the tether. We show that the tether affects the magnitude of the correlations which can be used in a measurement to probe the properties of an attached tethering substance. For the configurations of current interest using micron scale cantilevers in water, we show that the magnitude of the fluid coupling between the cantilevers is sufficiently small such that the influence of the tether can be significant. Our results show that the cross-correlation is more sensitive to tether stiffness than the auto-correlation indicating that a two-cantilever measurement has improved sensitivity when compared with a measurement using a single cantilever. (C) 2014 AIP Publishing LLC.
\end{abstract}

[http://dx.doi.org/10.1063/1.4900525]

\section{INTRODUCTION}

There is broad scientific and technological interest in understanding the correlated motion of small elastic objects in a viscous fluid. ${ }^{1-7}$ In many situations, it is desirable for the oscillating objects to be strongly coupled through the fluid motion. For example, the coupled motion of cilia and flagella in microorganisms is essential for important biological processes such as transport and locomotion; ${ }^{8-10}$ and the correlated motion of micron scale beads held in optical traps in a viscous fluid is central to current microrheological techniques that probe the material properties of complex fluids with unprecedented spatial and temporal precision. ${ }^{5}$ In many sensing applications, it is desirable to minimize the coupling between the oscillating objects due to the fluid in order to probe quantitatively the effect of other interactions that are present. ${ }^{4,11-13}$ For example, the dynamics of the oscillating objects could also be correlated due to a direct tethering by a biomolecule or bulk material of interest. However, it has emerged in the literature that the coupling due to the viscous fluid can be quite complicated and subtle with the correlated dynamics depending upon the object's geometry, separation, amplitude of oscillation and oscillation frequency as well as the rheological properties of the surrounding fluid. ${ }^{1,3,5,7,13}$ In order to effectively probe the properties of a direct tether between the objects it is necessary to have a quantitative understanding of the underlying fluid coupling for the precise conditions of interest.

In this paper, we are interested in sensing type applications in a viscous fluid where the dynamics and properties of a tethered biomaterial or elastic object is of interest. Our approach is to use numerical simulations for the conditions of experiment to quantify the coupled dynamics of two elastic objects in a viscous fluid that are also elastically tethered directly together. It is well known that the dynamics of biomolecules are described by extremely rich and complex energy landscapes. ${ }^{14,15}$ One way to represent the mechanical properties of biomolecules are through measurements of elasticity and damping. For example, the mechanical properties of a polymer or protein can be described by treating the biomolecule as a viscoelastic material. ${ }^{13,16-18}$ This approach yields an effective molecular stiffness $k_{\mathrm{mol}}$, representing the elastic contribution, and an effective molecular damping $\gamma_{\text {mol }}$, representing the dissipative contribution, for the single biomolecule of interest.

As an example, Meiners and Quake ${ }^{11}$ explored the dynamics of two micron scale beads in fluid that were also tethered together by a strand of DNA. This approach achieved femtonewton force resolution on millisecond time scales and shed new physical insights into the complex elastic properties and internal hydrodynamic interactions of DNA. Extending this approach to a pair of micron or nanoscale cantilevers would provide access to piconewton forces on microsecond time scales ${ }^{4,12}$ which is of significant biological relevance. ${ }^{19-21}$ Recent experimental measurements used a pair of micron scale cantilevers to probe the mechanical properties of a single molecule of dextran tethered between the cantilever tips. ${ }^{13}$ Using a simple harmonic oscillator model it was possible to extract a molecular stiffness and molecular damping for a dextran molecule. ${ }^{13}$

A significant question that we explore is under what conditions the hydrodynamic coupling between two micron scale cantilevers in water can be made small enough, in a 
practical situation of interest, where the dynamics of a soft elastic tether can be probed. In the absence of a direct tether connecting the cantilevers there has been significant progress in building a physical understanding of the correlated dynamics. Paul and Cross ${ }^{2,22}$ used the fluctuation-dissipation theorem to compute the stochastic dynamics of an array of objects for the precise conditions of experiment using only deterministic calculations. This approach has been validated by direct comparison with experimental measurements. ${ }^{6,13}$

In this paper, we extend this approach to include an elastic tether between the two cantilevers for the conditions of experiment. In our numerical simulations, we represent the elastic tether as a spring force with a stiffness $k$ that is meant to represent the elastic contribution of a tethered biomolecule. In the simulations, the positions of the bases of the two cantilevers are fixed and our results represent the stochastic dynamics of the system at equilibrium. We directly compare our theoretical calculations with experimental measurement in the absence of a tether for experimental conditions similar to those of Ref. 13. We then use the numerical approach to probe the dynamics of the cantilever pair with an elastic tether to build a physical understanding of the dynamics and to quantify the performance capabilities of this approach. In our study, it is not our intention to predict the correlated dynamics of the cantilevers for a specific biomolecule or bulk material with a complex energy landscape, but rather to demonstrate the potential of this approach to probe the dynamics of an elastic tether in the presence of a viscous fluid.

\section{APPROACH}

The geometry and configuration of the microcantilever pair is shown in Fig. 1. Each cantilever is identical and has a length $L$, height $h$, and width $W$. The cantilevers are separated from one another by a distance $d$. Each cantilever has a pyramidal tip located near its distal end. The two cantilevers are positioned such that their ends overlap by a distance of $L_{1}$. The base of the pyramidal tip that is attached to the surface of the cantilever has a square cross-section with a side length of $L_{t}$. The height of the pyramid is $h_{t}$. In our numerical simulations, we have modeled the pyramidal tips as rectangular prisms with a square base of length $L_{t}$ and a height of $h_{t}$. We found this approximation to be sufficient as expected for low Reynolds number flows. One could include the added complexity of the actual pyramid geometry if desired although this is not something we explored. The center of the cantilever tips are located $4 \mu \mathrm{m}$ from the distal edge of the cantilever and is centered upon the width of the cantilever. The shortest distance between the tips of the two cantilevers is $s$. The solid black line connecting the two tips represents a Hookean tether of spring constant $k_{t}$. In our numerical simulations this tethering is represented only as a force acting on the two cantilevers.

In experiments, the cantilevers are usually composed of silicon nitride where the precise stoichiometry is unknown. We model the cantilevers using an effective Youngs modulus $E$ and an effective density $\rho_{c}$. These effective values were determined numerically by comparing numerical results with experimental measurement of the auto-correlation of equilibrium fluctuations

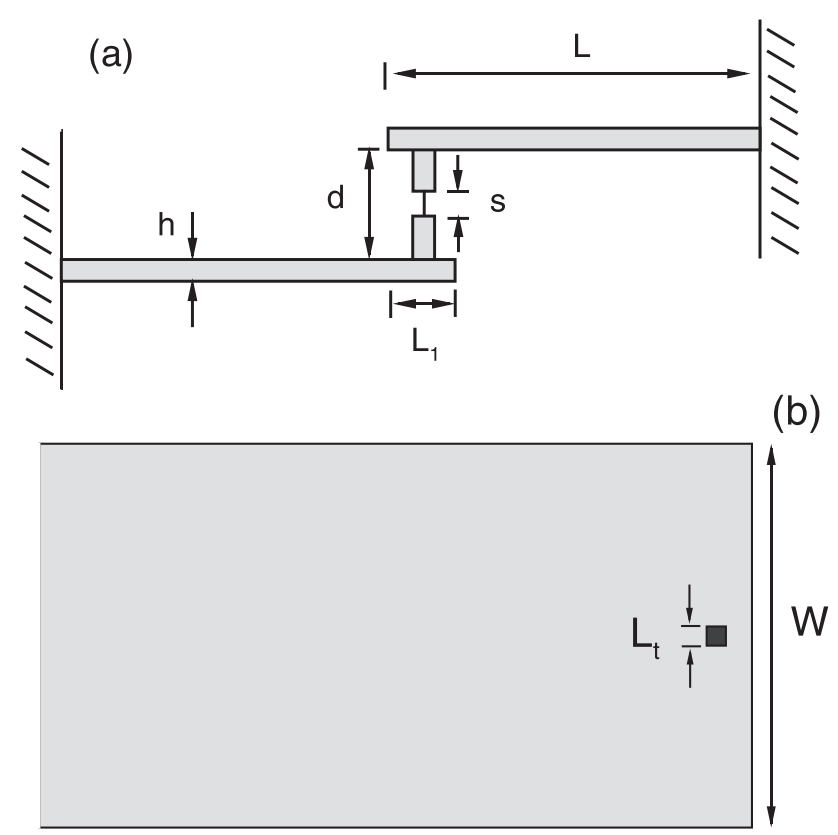

FIG. 1. Two micron scale cantilevers immersed in a viscous fluid (not drawn to scale). (a) Two identical cantilevers of length $L$, width $W$, and height $h$ are immersed in a viscous fluid and brought within close proximity of one another. The two cantilevers are separated by a distance $d$. Each cantilever has a rectangular tip located near its distal end with a separation between the tips of $s$. The distal ends of the two cantilevers have an overlap distance of $L_{1}$. The two cantilevers are tethered to one another by a Hookean spring with a spring constant of $k_{c}$. In this figure, the tether is represented as a vertical solid line between the two tips. In the numerical simulations the tether is represented as Hookean spring force with a spring constant of $k_{t}$ acting on the tips of the two cantilevers. (b) A close-up view, from above, near the distal end of one of the cantilevers. The cantilever has a width $W$ and the dark region is the rectangular tip which has a square base of side-length $L_{t}$ and height $H_{t}$.

in cantilever displacement for a single cantilever immersed in water. The effective spring constant of a cantilever is $k_{c}$ and the resonant frequency of the fundamental mode of transverse oscillation in vacuum is $\omega_{0}$. The cantilevers are immersed in room temperature water with a dynamic viscosity $\mu_{f}$ and density $\rho_{f}$. The numerical values of all of the parameters for our specific configuration are given in Table I.

Several important nondimensional parameters are given in Table II that provide further insight into the physical

TABLE I. Parameters describing the array of two micron scale cantilevers shown in Fig. 1. The cantilevers are identical with length $L$, width $W$, and height $h$ and have an effective Young's modulus $E=110.8 \mathrm{GPa}$ and density $\rho_{c}=5344 \mathrm{~kg} / \mathrm{m}^{3}$. The resonant frequency of the fundamental mode of the cantilever in vacuum is $\omega_{0}$ and the effective spring constant of the cantilever is $k_{c}$. The separation between the cantilevers is $d=6.7 \mu \mathrm{m}$ and between the tips is $s=879 \mathrm{~nm}$. The distal ends of the two cantilevers overlap one another by a distance $L_{1}=8 \mu \mathrm{m}$. Each cantilever has a pyramidal tip near its distal end which is modeled as a rectangular prism with a square base of length $L_{t}=2 \mu \mathrm{m}$ and height $h_{t}=2.9 \mu \mathrm{m}$. The center of the tip is located a distance of $4 \mu \mathrm{m}$ from the distal edge of the cantilever and is centered upon the cantilever's width. The cantilevers are immersed in room temperature water with density $\rho_{f}=997.5 \mathrm{~kg} / \mathrm{m}^{3}$ and dynamic viscosity $\mu_{f}=9.41 \times 10^{-4} \mathrm{~kg} / \mathrm{m}-\mathrm{s}$.

\begin{tabular}{lcccc}
\hline \hline $\begin{array}{l}L \\
(\mu \mathrm{m})\end{array}$ & $\begin{array}{c}W \\
(\mu \mathrm{m})\end{array}$ & $\begin{array}{c}h \\
(\mu \mathrm{m})\end{array}$ & $\begin{array}{c}k_{c} \\
(\mathrm{~N} / \mathrm{m})\end{array}$ & $\begin{array}{c}\omega_{0} \\
(\mathrm{rad} / \mathrm{s})\end{array}$ \\
\hline 185 & 40.5 & 0.8 & 0.096 & $1.22 \times 10^{5}$ \\
\hline \hline
\end{tabular}


regime of the dynamics. For a cantilever oscillating in a fluid the frequency parameter $R_{0}$ is given by

$$
R_{0}=\frac{\omega_{0} W^{2}}{4 \nu_{f}}
$$

and represents the ratio of local inertial forces to viscous forces, where $\nu_{f}=\mu_{f} / \rho_{f}$ is the kinematic viscosity. The frequency parameter plays the role of a frequency based Reynolds number. A value of $R_{0} \approx 50$ indicates the relative importance of local inertial forces and is a typical value for micron scale cantilevers in water. We point out that this is in contrast to the experiment of Meiners and Quake ${ }^{11}$ where two micron scale beads in water were held in optical traps which yielded $R_{0} \ll 1$. In the limit of vanishing frequency parameter the fluid dynamics are described by the Stokes equations where analytical solutions are available. ${ }^{23}$ In our case, fluid inertia is significant and the flow interactions between the cantilever tips is three-dimensional which makes analytical progress very difficult and a numerical approach appealing.

The mass loading parameter $T_{0}$ is given by

$$
T_{0}=\frac{\pi}{4} \frac{\rho_{f} W}{\rho_{c} h},
$$

and represents the ratio of the mass of a cylinder of fluid with a radius of $W / 2$ to the mass of the cantilever. A value of $T_{0} \approx 7$ is indicative of the importance of the fluid mass that is also oscillating along with the cantilever. The length scale of the unsteady viscous boundary layer ${ }^{24}$ is proportional to the Stokes length $\delta_{0}=\sqrt{\nu_{f} / \omega_{0}}$. For our configuration $\delta_{0} / d=0.4$ which indicates that there will be viscous interactions between the cantilevers. The magnitude of the deflection of the cantilever due to Brownian motion, in the absence of the tether, can be estimated directly from the equipartition of energy theorem. ${ }^{25}$ The root-mean-square value of the displacement of the cantilever at its distal end is $x_{\mathrm{rms}}=\left\langle x^{2}\right\rangle^{1 / 2}=\sqrt{k_{B} T / k_{c}}$, where $k_{B}$ is Boltzmann's constant, $T$ is the temperature, $x$ is the displacement of the distal end of the cantilever, and \langle\rangle indicates an equilibrium ensemble average. At room temperature $T=300 \mathrm{~K}$ this yields $x_{\mathrm{rms}}=0.21 \mathrm{~nm}$, where $x_{\mathrm{rms}} / s \ll 1$ indicating that the magnitude of the thermally induced vibrations is much smaller than the tip to tip separation between the cantilevers. Thus, we model the case where the increase in extension is small,

TABLE II. Nondimensional parameters describing the dynamics of an array of cantilevers immersed in a viscous fluid: $R_{0}$ is the frequency parameter of the cantilever evaluated at the resonant frequency of oscillation in a vacuum, $T_{0}$ is the mass loading parameter, $\xi=k_{t} / k_{c}$ is the stiffness ratio of the tether spring constant to the cantilever spring constant, $\delta_{0}=2.8 \mu \mathrm{m}$ is the thickness of the unsteady viscous boundary layer, and the root-mean-squared value of the cantilever deflections due to Brownian motion in the absence of a direct tether is $x_{\mathrm{rms}}=0.21 \mathrm{~nm}$. The Stokes length has been scaled by the cantilever separation $d$ and the root-mean-square deflection of the cantilever has been scaled by the tip-to-tip separation $s$.

\begin{tabular}{lcccc}
\hline \hline$R_{0}$ & $T_{0}$ & $\xi$ & $\delta_{0} / d$ & $x_{\text {rms }} / s$ \\
\hline 53.0 & 7.4 & $0 \leq \xi \leq 0.95$ & 0.4 & $2 \times 10^{-3}$ \\
\hline \hline
\end{tabular}

which justifies the representation of the tether as a simple spring.

An estimate of the magnitude of the Brownian forces acting on an individual cantilever is given by $F_{11}=k_{c} x_{\text {rms }}$ $\approx 20 \mathrm{pN}$. We will show that the magnitude of the correlated force of the Brownian motion for the cantilever pair of our configuration is an order of magnitude smaller at $F_{12}$ $\approx 2 \mathrm{pN}$. The time resolution of the cantilever pair can be estimated from the resonant frequency of the fundamental mode of oscillation of a cantilever in fluid $\omega_{f}$. For typical micron scale cantilevers used in atomic force microscopy (AFM) the resonant frequency is reduced by approximately a third when placed in water such that $\omega_{f} \approx \omega_{0} / 3$. This yields a time resolution of $\tau \approx 2 \pi / \omega_{f} \approx 0.2 \mathrm{~ms}$. Therefore, using two typical, and readily available, micron scale cantilevers in water the cantilever pair has piconewton force resolution with a time resolution of less than a millisecond. We note that this resolution could be improved significantly, if desired, by tailoring the cantilever geometry and material properties. However, a significant experimental advantage of micron scale cantilevers for use in water is that the quality factor of the oscillations is typically larger than unity and results in a well defined resonance peak. This is in direct contrast to nanoscale cantilevers which are typically strongly overdamped in water. ${ }^{2}$

It is convenient to represent the magnitude of the spring constant of the tether $k_{t}$ as the nondimensional stiffness ratio $\xi=k_{t} / k_{c}$, where $\xi=0$ is the special case of no tether and $\xi=1$ is the special case where the spring constants of the cantilevers and the tethering are identical. For many biosensing and materials applications one is interested in probing the limit of a soft tether, where $\xi \ll 1$. The limit of $\xi \gg 1$ is also of interest and could be used, for example, to determine the presence or absence of a tethered object although this is not something we have explored.

The dynamics of the cantilevers can be described using the methods of continuum mechanics. Using the relevant length scales in the problem we can construct a Knudsen number $\mathrm{Kn}=\lambda / s$, where $\lambda$ is the approximate size of a water molecule. This yields $\mathrm{Kn} \approx 1 \times 10^{-5} \ll 1$ indicating molecular effects are negligible. As pointed out by Ekinci et al., ${ }^{26}$ quantifying the ratio of relevant time scales is insightful and has been used to demonstrate universal features in oscillating flows. For the configuration explored here the Weissenberg number $\mathrm{Wi}=\omega_{0} \tau$, where $\tau \approx 1 \times 10^{-12} \mathrm{~s}$ is the characteristic time between collisions for a water molecule. Using these values yields $\mathrm{Wi} \approx 1 \times 10^{-7} \ll 1$ which also indicates that molecular effects will be negligible.

\section{A. Theory}

We use the approach of Paul and Cross ${ }^{2}$ to compute the stochastic dynamics of a pair of cantilevers immersed in a viscous fluid and with a direct tether. We refer the reader to Ref. 22 for the specific details of the underlying approach and we only provide the essential details here. The essence of the approach is to initially perturb the cantilever pair and to then compute deterministically the return to equilibrium. Using the fluctuation-dissipation theorem, we quantitatively relate how 
the cantilevers return to equilibrium to the stochastic dynamics of the cantilevers when driven by Brownian motion.

In the distant past, we apply a force of constant magnitude $F_{0}$ to the distal end of one of the cantilevers. For concreteness, we apply this force to the lower cantilever of Fig. 1 which we will refer to as cantilever 1 . We will refer to the upper cantilever as cantilever 2 , although this numbering is arbitrary. At time $t=0$, the force is removed and the cantilevers return to equilibrium. The deterministic displacement of the distal ends of the two cantilevers at time $t$ are $X_{1}(t)$ and $X_{2}(t)$, respectively. The ring down of cantilever 1 can be used to obtain the auto-correlation of equilibrium fluctuations in cantilever displacement from

$$
\left\langle x_{1}(0) x_{1}(t)\right\rangle=k_{B} T \frac{X_{1}(t)}{F_{0}} .
$$

We emphasize that in our notation an upper case $X(t)$ is used for the deterministic displacement and a lower case $x(t)$ is used for the stochastic displacement where the displacements are positive in the upward direction in Fig. 1. Similarly, the cross-correlations in equilibrium fluctuations of cantilever displacement can be computed from $X_{2}(t)$ as

$$
\left\langle x_{1}(0) x_{2}(t)\right\rangle=k_{B} T \frac{X_{2}(t)}{F_{0}} .
$$

We point out that at time $t=0$ both of the cantilevers have some deflection. Cantilever 1 is deflected due to the force $F_{0}$ that has been applied to its distal end in the distant past and cantilever 2 is deflected since it is connected to cantilever 1 by the tether between the tips of the two cantilevers.

The fundamental mode of transverse oscillation of each cantilever can be described as a lumped mass with a spring constant $k_{c}$. The tether is a Hookean spring between these two lumped masses. It is then straight forward to determine the initial displacements of the two cantilevers $X_{1}(0)$ and $X_{2}(0)$ which can be used in Eqs. (3) and (4) to yield the following relationships for the zero time-lag correlation functions:

$$
\begin{aligned}
\left\langle x_{1}(0) x_{1}(0)\right\rangle & =\frac{k_{B} T}{k_{c}}\left(\frac{1+\xi}{1+2 \xi}\right), \\
\left\langle x_{1}(0) x_{2}(0)\right\rangle & =\frac{k_{B} T}{k_{c}}\left(\frac{\xi}{1+2 \xi}\right) .
\end{aligned}
$$

In the limit of no tethering $\xi=0$ the auto-correlation of equilibrium fluctuations becomes $x_{\mathrm{rms}}^{2}$ and the cross-correlation vanishes as expected. We point out that the correlation functions at zero time lag can be used to yield a value of the spring constant of an attached tether of unknown stiffness from an experimental measurement.

Once the correlations are known, the spectral properties can be computed using a Fourier transform of Eqs. (3) and (4). The noise spectrum of the auto-correlated dynamics $G_{11}$ is given by ${ }^{22}$

$$
G_{11}(\omega)=4 \int_{0}^{\infty}\left\langle x_{1}(0) x_{1}(t)\right\rangle \cos (\omega t) d t
$$

Similarly, the noise spectrum of the cross-correlated dynamics $G_{12}$ is given by

$$
G_{12}(\omega)=4 \int_{0}^{\infty}\left\langle x_{1}(0) x_{2}(t)\right\rangle \cos (\omega t) d t .
$$

The deterministic quantities $X_{1}(t)$ and $X_{2}(t)$ can be computed using analytical models or numerical simulations. We use full three-dimensional and time-dependent finite element numerical simulations ${ }^{27}$ for the conditions of experiment to compute $X_{1}(t)$ and $X_{2}(t)$. In our numerical approach we numerically simulate the incompressible Navier-Stokes equations that are coupled with the full equations of elasticity. More details regarding the finite element approach used can be found in Refs. 22 and 28.

In our numerical simulations, we apply an upward force of magnitude $F_{0}$ to cantilever 1 and allow the system to reach equilibrium. We then remove the force and allow the system to return to equilibrium. The motion of the distal ends of the cantilevers as the system returns to equilibrium are precisely $X_{1}(t)$ and $X_{2}(t)$. We then compute the correlation functions using Eqs. (3) and (4) and the noise spectra using Eqs. (7) and (8). We have performed numerous numerical tests of spatial and numerical convergence to ensure the accuracy of our numerical results.

\section{B. Experiment}

The experiment follows the correlation force spectroscopy approach described in Refs. 6 and 13. In our case, the two cantilevers are vertically offset from one another in order to improve measurement sensitivity of a tethered object. In our configuration, the motion of the cantilevers translates directly to compression and tension of the tethered object. In addition, our cantilevers have a significant overlap distance (see $L_{1}$ in Fig. 1) and each cantilever has a pyramidal tip attached to its distal end.

Each cantilever is identical and is a commercially available AFM cantilever (Bruker ORC8-W, Cantilever B). The top cantilever in Fig. 1 is mounted in an atomic force microscope (Asylum Research, MFP3D-bio, CA). A custom built cantilever mount is incorporated in place of the typical AFM sample upon which we mounted the lower cantilever of Fig. 1. For the lower cantilever, a laser (Schäfter and Kirchhoff GmbH, Hamburg, Germany, 51 nanoFCM) is reflected off its surface and onto a split photodiode (Pacific Silicon Sensor, CA, QP50-6-18 u-SD2). A different wavelength laser is used for each cantilever, $680 \mathrm{~nm}$ for the bottom cantilever and $860 \mathrm{~nm}$ for the top cantilever. This allows for frequency filtering and prevents optical contamination between the signals. The signals of the cantilevers are recorded synchronously using an Asylum Research AFM controller with a Nyquist frequency of $25 \mathrm{kHz}$.

Prior to making any measurements, the cantilever pair is immersed in fluid and the system is allowed to reach thermal equilibrium for about $40 \mathrm{~min}$. During a typical measurement a time sequence of voltages are collected from the diodes which are then multiplied by a calibration constant to yield the stochastic cantilever deflections $x_{1}(t)$ and $x_{2}(t)$. Using 
Fourier methods, ${ }^{6}$ we then compute the correlation functions and noise spectra from the cantilever deflections.

\section{RESULTS}

We first quantify the dynamics of the two-cantilever array in the absence of a tether $\xi=0$. This serves several purposes. First, we use this to validate our theoretical approach by directly comparing our results with experimental measurements. Second, this allows us to quantify the dynamics, and in particular, the amount of coupling between the cantilevers due to the surrounding viscous fluid. The fluid coupling represents the amount of background coupling that must be overcome in order to detect the presence of any tethering.

Figure 2 shows a direct comparison between our numerical simulations and experimental measurements. The solid lines are results from numerical simulation and the data symbols are experimental measurement. Overall, the agreement is excellent. Figure 2(a) shows the auto-correlation in equilibrium fluctuations in cantilever displacement. The general shape of the auto-correlation function is typical of micron scale AFM cantilevers in fluid. ${ }^{22}$ Although we do not show it here the presence of the second cantilever has very little affect upon the auto-correlation.

Figure 2(b) shows the cross-correlation in equilibrium fluctuations in cantilever displacement. As expected, there are regions of both correlated and anticorrelated dynamics which can be connected with the interplay between the viscous and potential contributions to the fluid dynamics. ${ }^{7}$ It is interesting to note that the shape of the cross-correlation curve is quite different than what has been found for the case of two micron scale cantilevers that are separated along their axial direction ${ }^{6,22}$ and for two cantilevers that are vertically separated but without any overlap. ${ }^{12}$ For small time lags, Fig. 2(b) shows that the dynamics are anticorrelated as indicated by the negative values of the cross-correlation. We explored this in some detail and determined that this behavior is due to the significant overlap of the two cantilevers given by $L_{1}$.

Our results indicate that full finite element numerical simulations for the precise geometry of interest can achieve excellent quantitative agreement with experimental measurements. This suggests that using currently available finite element algorithms it is possible to numerically explore the stochastic dynamics of elastic objects for the sophisticated geometries and situations of interest to the development of new technologies.

Given the excellent agreement between the experiments and numerics, we next proceed to explore numerically the dynamics of a cantilever pair with a tether. Our intention is to quantify the expected performance over a range of conditions that could be used to guide future experiments that also include a tethering substance. There are significant experimental challenges that must be overcome in order to tether an object of interest between the tips of the cantilevers. Our numerical investigation provides physical insights into the correlated dynamics that can be used to tailor future experimental efforts.

We have performed a series of 5 numerical simulations over a range of stiffness ratios, where $0 \leq \xi \leqq 1$ with the specific values $\xi=\{0,0.05,0.10,0.19,0.95\}$. One could imagine tethering a single soft biomolecule between the cantilevers to yield $\xi \ll 1$. An important question is what is the smallest value of $\xi$ that would yield dynamics where an experimental measurement can be used to characterize the tethering molecule. For example, the longitudinal spring constant of DNA under extension and for small elongation ${ }^{11}$ is $k_{\mathrm{DNA}} \approx 1 \times 10^{-6} \mathrm{~N} / \mathrm{m}$. If one were to tether a strand of DNA between the cantilevers this would yield $\xi \approx 1 \times 10^{-5}$ which is beyond the resolution of this instrument. However, it is possible to decrease the spring constant of the cantilever significantly by uniformly decreasing its dimensions ${ }^{2,29}$ which could make such a measurement possible. It is also possible to tether many molecules in parallel between the cantilever tips which would increase the effective value of $\xi$ as the number of tethered molecules increases.

In the experiments of Radiom et al. ${ }^{13}$ a single molecule of dextran was tethered between two micron scale cantilevers that were held in constant deflection. These experiments yielded a molecular stiffness of $k_{\text {dextran }} \approx 0.015 \mathrm{~N} / \mathrm{m}$ and a molecular damping of $\gamma_{\text {dextran }} \approx 2 \times 10^{-7} \mathrm{~kg} / \mathrm{s}$. Although these results are relevant to our theoretical study we point out that the experimental conditions are not precisely the same as the numerical simulations we discuss here. Namely, in the experiments the position of the cantilever bases are controlled such that the cantilevers are held in constant deflection and also the cantilevers undergo a small thermal drift during the measurement. In contrast, the positions of the cantilever bases in our simulations are stationary and the two cantilevers
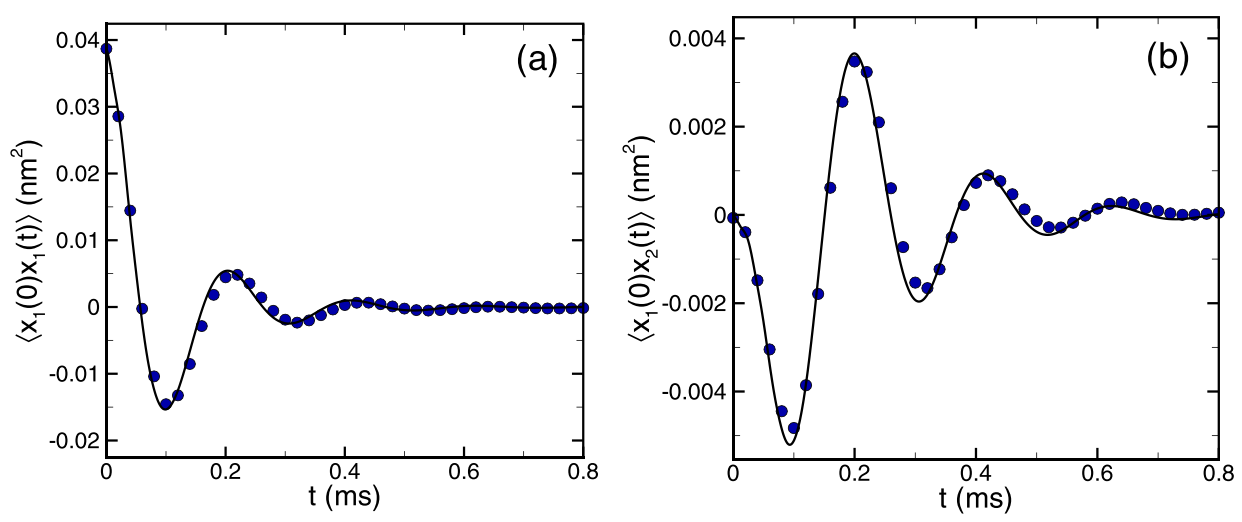

FIG. 2. The correlations of equilibrium fluctuations in tip displacement for two cantilevers immersed in water without the presence of a tether. The solid lines are results from numerical simulation and the data symbols are from experimental measurement: (a) autocorrelation, (b) cross-correlation. 

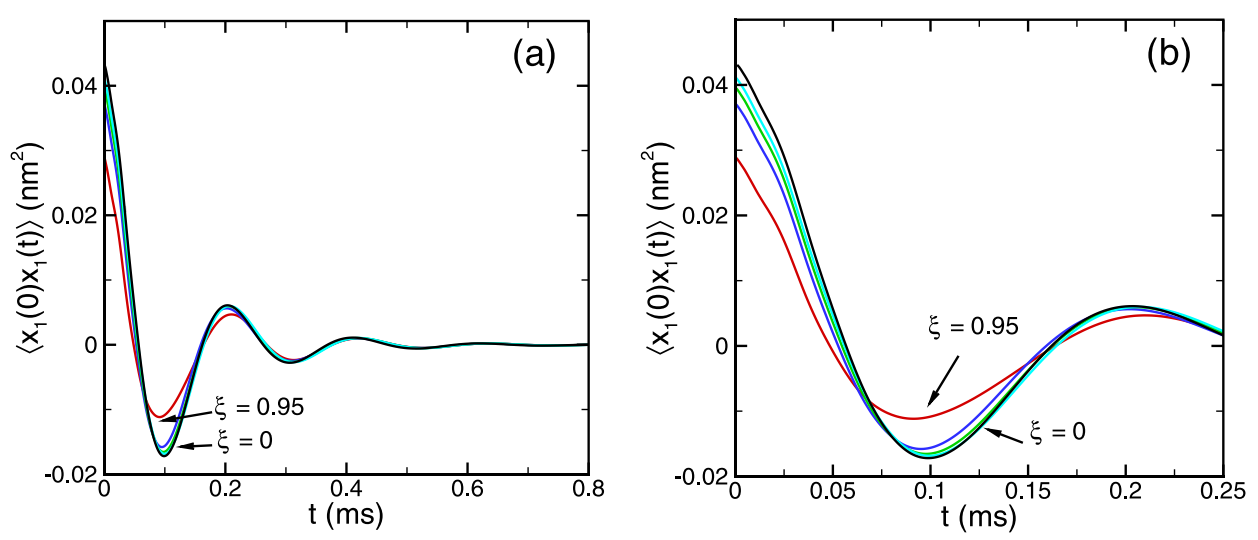

FIG. 3. The auto-correlation of equilibrium fluctuations in cantilever displacement over a range of different tethering ratio $\xi$. Curves are shown for five different values of $\xi$, where $\xi=$ $\{0$ (black), 0.05 (cyan), 0.10 (green), 0.19 (blue), 0.95 (red) $\}$. The curves for $\xi=0$ and $\xi=0.95$ are labelled with the remaining curves in sequence. (a) The complete auto-correlation. (b) A close up view of the autocorrelations for early times.

remain in equilibrium. In addition, we have not included a molecular damping in our simulations. Although it is not our intention to reproduce these experimental results using our numerical simulations it is important to point out that for these experiments $\xi=0.16$ which is in the range of the stiffness ratios explored here. As a result, our numerical simulations can be used to gain physical insights into the importance of the fluid coupling in these experiments.

In Fig. 3, we show numerical results for the autocorrelation of equilibrium fluctuations in cantilever displacement. In Fig. 3(a), it is evident that the presence of the tether has very little effect upon the auto-correlation, even for the case when the tether is nearly as stiff as a cantilever $\xi=0.95$. Figure 3 (b) shows a close-up view of the autocorrelation for small values of the time lag $t$ which allows the general trends to be observed more clearly. The zero time lag value of the auto-correlation agrees with the predictions of Eq. (5). As expected, the value of the autocorrelation at zero time lag decreases with increasing values of the stiffness ratio. For the smaller values of the stiffness ratio the auto-correlation results are nearly identical with the results in the absence of tethering $\xi=0$.

The cross-correlation of equilibrium fluctuations in cantilever displacement are shown in Fig. 4. The cross-correlations

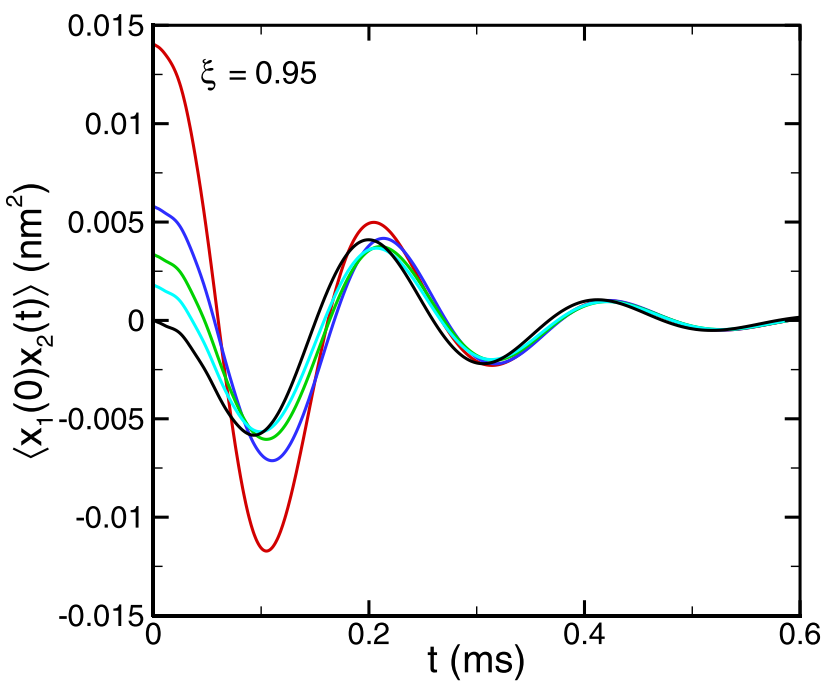

FIG. 4. The cross-correlation of equilibrium fluctuations over a range of tether ratios $\xi=\{0$ (black), 0.05 (cyan), 0.10 (green), 0.19 (blue), 0.95 (red) $\}$. The curve for $\xi=0.95$ is labelled with the remaining curves occurring in order. are more sensitive to the presence of the tether when compared with the auto-correlations of Fig. 3. The value of the cross correlation for zero time lag agrees with prediction given by Eq. (6). As expected, the value of the crosscorrelation at zero time lag vanishes in the absence of tethering $\xi=0$. The presence of the tethering between the cantilevers results in a positive correlation at small time lags in contrast to the anti-correlated dynamics found at small time lags without the tether. In this case, the positive correlation due to the direct tethering is overcoming the anticorrelated contribution from the fluid dynamics. Overall, the effect of the tethering is most significant for small time lags and for larger time lags $t \gtrsim 0.2$ the effect of the tethering is minimal. The presence of the tethering affects the magnitude of both the auto and crosscorrelations with little effect upon the phase of the curves.

The noise spectra of the dynamics are shown in Fig. 5. Figure 5(a) illustrates the noise spectrum of the autocorrelated motion $G_{11}(\omega)$. The frequency has been normalized by the resonant frequency in vacuum $\omega_{0}$ to illustrate the significant reduction in frequency due to the fluid. The magnitude of the noise spectrum is affected slightly by the presence of the tether. As expected, the magnitude of the cantilever motion decreases with increasing values of the stiffness ratio for fixed cantilever stiffness. For smaller values of the stiffness ratio $\xi \lesssim 0.10$ the noise spectrum is nearly unchanged.

The noise spectrum of the cross-correlated motion $G_{12}$ is shown in Fig. 5(b). The magnitude of the cross-correlated noise spectrum increases with increasing values of the stiffness ratio. In general, the noise spectrum is more sensitive to the presence of the tether than $G_{11}$.

Although an analytical description of the threedimensional fluid-solid interactions for this problem is not available, it is possible to gain some physical insight into the dynamics using ideas from a normal mode analysis. ${ }^{7,13}$ Using a normal mode analysis the dynamics of the two cantilevers can be decomposed into a symmetric and antisymmetric mode. In the symmetric mode, the two cantilevers oscillate in phase where the separation between the tips would remain constant at all times and therefore, the tether is never stretched or compressed. In the antisymmetric mode, the oscillations of the two cantilevers are exactly out of phase with respect to one another and the tether experiences both tension and compression.

Each normal mode will have a peak associated with it in Fig. 5(b), where the magnitude and width of the peak 

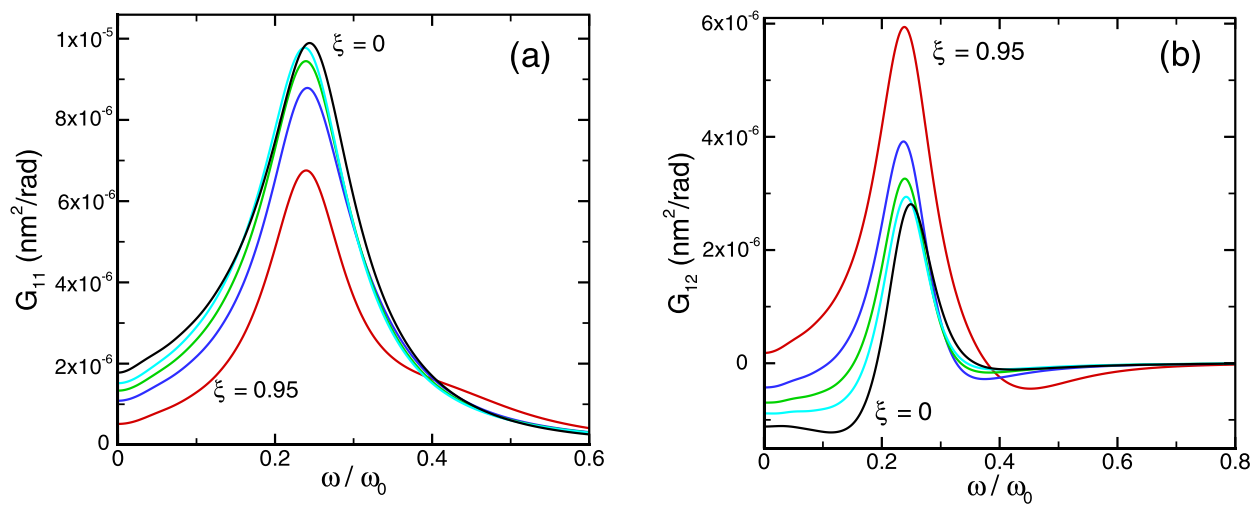

FIG. 5. The noise spectra for $\xi=$ $\{0$ (black), 0.05 (cyan), 0.10 (green), 0.19 (blue), 0.95 (red) $\}$. The curves for $\xi=0$ and $\xi=0.95$ are labelled with the remaining curves occurring in sequence. (a) The noise spectrum of the auto-correlated motion $G_{11}(\omega)$. (b) The noise spectrum of the crosscorrelated motion $G_{12}(\omega)$.

depends upon the frequency dependent damping and mass loading of the viscous fluid. Both the mass and internal damping of the cantilever are much less significant in comparison to that of the fluid. In the limit of very high frequency oscillations the dynamics become potential and viscous effects vanish. ${ }^{7}$ In the potential dynamics limit, the peaks become delta functions with a well defined frequency where the frequency of the antisymmetric mode is larger than that of the of symmetric mode.

Some of these features are evident in Fig. 5(b). The symmetric mode yields positive values of $G_{12}(\omega)$ and is responsible for the large positive peak. The antisymmetric mode yields negative values of $G_{12}(\omega)$ and is responsible for the negative peak in Fig. 5(b) at larger frequencies for $\xi \gtrsim 0.3$. As the stiffness ratio increases the symmetric mode becomes larger and, in the limit of a rigid tether between the cantilevers, the motion would be entirely composed of the symmetric mode. For values of the stiffness ratio $\xi \gtrsim 1$, Fig. 5(b) shows that the dynamics are dominated by the symmetric mode. For small values of the stiffness ratio, both the antisymmetric and symmetric modes are significant. In this light there is a signature of the tether present in Fig. 5(b) where the tether increases the magnitude of $G_{12}(\omega)$ while also the symmetric mode gains dominance.

\section{CONCLUSION}

We have quantified the correlated dynamics of a pair of micron scale cantilevers immersed in a viscous fluid that are also elastically tethered to one another by a spring force applied between the cantilever tips to represent the molecular stiffness of the tethered material. In the absence of the elastic tether, we have validated our theoretical approach with a direct comparison to experimental measurements with excellent agreement. This demonstrates that deterministic finite element numerical simulations can be used to compute the stochastic dynamics of a cantilever array for the precise conditions of experiment. This includes complex features of the cantilever geometry and configuration such as the pyramidal cantilever tips and cantilever overlap. The numerical computations, even at this complexity, can be performed on single workstation and do not require supercomputing resources or excessive computational times.

Using our numerical approach, we gained new physical insights into the dynamics of a tethered cantilever pair that is of current experimental interest. Our results allowed us to explore the performance characteristics of this system over a range of parameters. Our results demonstrate that the crosscorrelations are more sensitive than the auto-correlations to the presence of an elastic tether. This suggests that the two cantilever approach will provide greater resolution of molecular properties than the single cantilever approach. The elastic tether effectively modifies the magnitude of the correlations with its most significant effect at small values of the time lag. Using a normal mode description, the dynamics of the symmetric and antisymmetric modes can be elucidated by the noise spectrum of the correlated motion. Our results demonstrate that it is possible to measure significant correlations due to the elastic tether even in the presence of the viscous fluid coupling.

Using our approach, it would be interesting to include more complex and realistic models of the tether between the cantilevers. This could include more sophisticated molecular stiffness models, molecular damping, and inertial contributions. The ability of microscale cantilevers to probe piconewton forces at millisecond time scales in fluid holds significant promise for the development of future technologies. Using numerical computations for the conditions of experiment, we gain new physical insights that are crucial in guiding these efforts.

\section{ACKNOWLEDGMENTS}

This research was supported by NSF Grant No. CBET0959228 and by Virginia Tech. We have also had many fruitful discussions with Dongjin Seo and Chris Honig.

${ }^{1}$ J. C. Meiners and S. R. Quake, "Direct measurement of hydrodynamic cross correlations between two particles in an external potential," Phys. Rev. Lett. 82(10), 2211-2214 (1999).

${ }^{2}$ M. R. Paul and M. C. Cross, "Stochastic dynamics of nanoscale mechanical oscillators in a viscous fluid," Phys. Rev. Lett. 92(23), 235501 (2004).

${ }^{3} \mathrm{~S}$. Basak and A. Raman, "Hydrodynamic coupling between micromechanical beams oscillating in viscous fluids," Phys. Fluids 19, 017105 (2007).

${ }^{4}$ J. L. Arlett, M. R. Paul, J. Solomon, M. C. Cross, S. E. Fraser, and M. L. Roukes, "BioNEMS: Nanomechanical devices for single-molecule biophysics," Lect. Notes Phys. 711, 241-270 (2007).

${ }^{5}$ T. M. Squires and T. G. Mason, "Fluid mechanics of microrheology," Annu. Rev. Fluid Mech. 42, 413-438 (2010).

${ }^{6}$ C. D. F. Honig, M. Radiom, B. A. Robbins, J. Y. Walz, M. R. Paul, and W. A. Ducker, "Correlations between the thermal vibrations of two cantilevers: Validation of deterministic analysis via the fluctuation-dissipation theorem," Appl. Phys. Lett. 100, 053121 (2012).

${ }^{7}$ M. R. Paul, M. T. Clark, and M. C. Cross, "Coupled motion of microscale and nanscale elastic objects in a viscous fluid," Phys. Rev. E 88, 043012 (2013). 
${ }^{8}$ A. Vilfan and F. Julicher, "Hydrodynamic flow patterns and synchronization of beating cilia," Phys. Rev. Lett. 96, 058102 (2006).

${ }^{9}$ E. Lauga and T. R. Powers, "The hydrodynamics of swimming microorganisms," Rep. Prog. Phys. 72, 096601 (2009).

${ }^{10}$ R. E. Goldstein, M. Polin, and I. Tuval, "Noise and synchronization in pairs of beating eukaryotic flagella," Phys. Rev. Lett. 103, 168103 (2009).

${ }^{11}$ J. C. Meiners and S. R. Quake, "Femtonewton force spectroscopy of single extended DNA molecules," Phys. Rev. Lett. 84(21), 5014-5017 (2000).

${ }^{12}$ M. T. Clark and M. R. Paul, "The stochastic dynamics of an array of atomic force microscopes in a viscous fluid," Int. J. Non Linear Mech. 42, 690-696 (2007).

${ }^{13}$ M. Radiom, C. D. F. Honig, J. Y. Walz, M. R. Paul, and W. A. Ducker, “A correlation force spectrometer for single molecule measurements under tensile load," J. Appl. Phys. 113, 013503 (2013).

${ }^{14}$ E. Evans, "Probing the relation between force-lifetime-and chemistry in single molecular bonds," Annu. Rev. Biophys. Biomol. Struct. 30, 105-128 (2001).

${ }^{15}$ H. Frauenfelder, S. G. Sligar, and P. G. Wolynes, "The energy landscapes and motions of proteins," Science 254, 1598-1603 (1991).

${ }^{16}$ M. Kawakami, K. Byrne, B. Khatri, C. B. Mcleish, S. Radford, and D. A. Smith, "Viscoelastic properties of single polysaccharide molecules determined by analysis of thermally driven oscillations of an atomic force microscope cantilever," Langmuir 20, 9299-9303 (2004).

${ }^{17}$ M. Dong, S. Husale, and O. Sahin, "Determination of protein structural flexibility by microsecond force spectroscopy," Nat. Nanotechnol. 4, 514-517 (2009).
${ }^{18}$ Y. Wang and G. Zocchi, "The folded protein as a viscoelastic solid," EPL 96, 18003 (2011).

${ }^{19} \mathrm{G}$. Bao and S. Suresh, "Cell and molecular mechanics of biological materials," Nature Mater. 2, 715-725 (2003).

${ }^{20}$ C. Bustamante, J. C. Macosko, and G. J. L. Wuite, "Grabbing the cat by the tail: manipulating molecules one by one," Nat. Rev. Mol. Cell Bio. 1, 130-136 (2000).

${ }^{21}$ H. Clausen-Schaumann, M. Seitz, R. Krautbauer, and H. E. Gaub, "Force spectroscopy with single bio-molecules," Curr. Opin. Chem. Biol. 4, 524-530 (2000).

${ }^{22}$ M. R. Paul, M. T. Clark, and M. C. Cross, "The stochastic dynamics of micron and nanoscale elastic cantilevers in fluid: Fluctuations from dissipation," Nanotechnology 17, 4502-4513 (2006).

${ }^{23}$ C. Pozrikidis, Boundary Integral and Singularity Methods for Linearized Viscous Flow (Cambridge University Press, Cambridge, 1992).

${ }^{24}$ L. Rosenhead, Laminar Boundary Layers (Oxford University Press, 1963).

${ }^{25} \mathrm{H}$.-J. Butt and M. Jaschke, "Calculation of thermal noise in atomic force microscopy," Nanotechnology 6, 1-7 (1995).

${ }^{26}$ K. L. Ekinci, D. M. Karabacak, and V. Yakhot, "Universality in oscillating flows," Phys. Rev. Lett. 101, 264501 (2008).

${ }^{27}$ CFD-ACE + solver, ESI CFD Headquarters, Huntsville, AL 25806, 2014.

${ }^{28}$ H. Q. Yang and V. B. Makhijani, "A strongly coupled pressure-based CFD algorithm for fluid-structure interaction,” AIAA Paper No. 94-0179, 1994, pp. 1-10.

${ }^{29}$ K. L. Ekinci and M. L. Roukes, "Nanoelectromechanical systems," Rev. Sci. Instrum. 76(6), 061101 (2005). 